Journal of Mathematics and Statistics 7 (2): 95-97, 2011

ISSN 1549-3644

(C) 2010 Science Publications

\title{
Complete Convergence of Exchangeable Sequences
}

\author{
George Stoica \\ Department of Mathematical Sciences, \\ University of New Brunswick, Saint John NB, Canada
}

\begin{abstract}
We prove that exchangeable sequences converge completely in the Baum-Katz sense under the same conditions as i.i.d. sequences do. Problem statement: The research was needed as the rate of convergence in the law of large numbers for exchangeable sequences was previously obtained under restricted hypotheses. Approach: We applied powerful techniques involving inequalities for independent sequences of random variables. Results: We obtained the maximal rate of convergence and provided an example to show that our findings are sharp. Conclusion/Recommendations: The technique used in the paper may be adapted in the similar study for identically distributed sequences.
\end{abstract}

Key words: Exchangeable sequences, rate of convergence, strong law of large numbers

\section{INTRODUCTION}

A sequence of random variables $\left\{\mathrm{X}_{\mathrm{n}}\right\}_{\mathrm{n} \geq 1}$ on the probability space $(\Omega, \mathrm{F}, \mathrm{P})$ is called exchangeable if for every n:

$\mathrm{P}\left[\mathrm{X}_{1} \leq \mathrm{X}_{1}, \ldots, \mathrm{X}_{\mathrm{n}} \leq \mathrm{X}_{\mathrm{n}}\right]=\mathrm{P}\left[\mathrm{X}_{\pi 1} \leq \mathrm{X}_{1}, \ldots, \mathrm{X}_{\pi \mathrm{n}} \leq \mathrm{X}_{\mathrm{n}}\right]$

for any permutation $\pi$ of $\{1,2, \ldots, n\}$ and any $\mathrm{x}_{\mathrm{i}} \in \mathrm{R}, \mathrm{i}=$ $1, \ldots, n$. In particular, exchangeable sequences are identically distributed and one can say that future samples behave like earlier samples, or any order of a finite number of samples is equally likely. Sampling without replacement, weighted averages of i.i.d. sequences, $\left\{\mathrm{Y}+\varepsilon_{\mathrm{n}}\right\}_{\mathrm{n} \geq 1}$ and $\left\{\mathrm{Y} . \varepsilon_{\mathrm{n}}\right\}_{\mathrm{n} \geq 1}$ are examples of exchangeable sequences, where $\left\{\varepsilon_{n}\right\}_{n \geq 1}$ are i.i.d. and independent of the random variable Y. By [Chow and Teicher, 2003, Theorem 7.3.3], called de Finetti's theorem, an exchangeable sequence $\left\{X_{n}\right\}_{n \geq 1}$ is conditionally i.i.d. given either the tail $\sigma$-field of $\left\{X_{n}\right\}_{n \geq 1}$ or the $\sigma$-field $G$ of permutable events.

\section{MATERIALS AND METHODS}

Under appropriate moment conditions, strong laws of large numbers for exchangeable sequences have been obtained in (Taylor and Hu, 1987; Etemadi and Kaminski, 1996; Etemadi, 2006; Rosalsky and Stoica, 2010). The rate of convergence in the above strong laws has not been obtained in full generality; for instance, papers (Zhao, 2004) assume $\mathrm{p}=2, \mathrm{r}=1$ and exponential, fourth and third order moments, respectively, for $\left\{X_{n}\right\}_{n \geq 1}$, whereas (Taylor and $\mathrm{Hu}, 1987)$ requires symmetry of the $\mathrm{X}_{\mathrm{n}} \mathrm{s}$ and obtains estimate (1) provided $p=2 r, 2 \leq p<4$. Using appropriate inequalities for independent sequences, the purpose of this study is to prove that exchangeable sequences converge completely in the Baum-Katz sense under the same conditions as i.i.d. sequences do.

\section{RESULTS AND DISCUSSION}

Theorem 1: Let $\left\{X_{n}\right\}_{n \geq 1}$ be a sequence of exchangeable random variables with $\mathrm{E}\left(\mathrm{X}_{1}\right)=0$ and $\mathrm{E}\left|\mathrm{X}_{1}\right|^{\mathrm{p}}<\infty$ for some $\mathrm{p} \geq 1$. If $0<\mathrm{r}<2, \mathrm{p} \geq \max \{\mathrm{r}, 1\}$ and $\mathrm{S}_{\mathrm{n}}:=\mathrm{X}_{1}+\ldots$ $+\mathrm{X}_{\mathrm{n}}$, then Eq. 1:

$$
\sum_{n=1}^{\infty} n^{p / r-2} P\left[\left|S_{n}\right| \geq n^{1 / r}\right]<\infty
$$

The following result (cf. (Petrov, 1995)) will be used in the proof of Theorem 1.

Lemma 2: Let $\left\{\xi_{n}\right\}_{n \geq 1}$ a sequence of independent random variables with $\mathrm{E}\left(\xi_{\mathrm{i}}\right)=0$ and $\mathrm{E}\left|\xi_{\mathrm{i}}\right|^{\mathrm{p}}<\infty$ for all $\mathrm{i} \geq$ 1 and some $\mathrm{p} \geq 1$. If $0<\mathrm{r}<2$ and $\mathrm{T}_{\mathrm{n}}:=\xi_{1}+\ldots+\xi_{\mathrm{n}}$, then:

$$
\begin{aligned}
& \mathrm{P}\left[\left|\mathrm{T}_{\mathrm{n}}\right| \geq \mathrm{n}^{1 / \mathrm{r}}\right] \leq \mathrm{C}_{\mathrm{n}}^{-\mathrm{p} / \mathrm{r}} \sum_{\mathrm{i}=1}^{\mathrm{n}} \mathrm{E}\left|\xi_{\mathrm{i}}\right|^{\mathrm{p}} \\
& \text { if } 1 \leq \mathrm{p} \leq 2 \text { (von Bahr }- \text { Esseen) } \\
& \mathrm{P}\left[\left|\mathrm{T}_{\mathrm{n}}\right| \geq \mathrm{n}^{1 / \mathrm{r}}\right] \leq \mathrm{C}_{\mathrm{n}}^{-\mathrm{p} / \mathrm{r}} \sum_{\mathrm{i}=1}^{\mathrm{n}} \mathrm{E}\left|\xi_{\mathrm{i}}\right|^{\mathrm{p}}+\mathrm{Cexp}\left(-\mathrm{Cn}^{2 / \mathrm{r}} \sigma^{-2}\right) \\
& \text { if } \mathrm{p} \geq 2 \text { (Fuk - Nagaev) }
\end{aligned}
$$


Where:

$$
\sigma^{2}=\sum_{i-1}^{n} E\left(\xi_{i}^{2}\right)
$$

Proof of Theorem 1: By [(Chow and Teicher, 2003), Corollary 7.3.5] there exists a regular conditional distribution $\mathrm{P}^{\omega}$ given the $\sigma$-field $\mathrm{G}$ such that for each $\omega \in \Omega$ the mixands $\left\{\xi_{n} \equiv \xi_{n}^{\omega}\right\}_{n} \geq 1$, i.e., the coordinate random variables of the Borel probability space $\left(\mathrm{R}^{\infty}, \mathrm{B}\left(\mathrm{R}^{\infty}\right), \mathrm{P}^{\omega}\right)$ are i.i.d. Namely, for all $\mathrm{n} \in \mathrm{N}$, any Borel function $\mathrm{f}: \mathrm{R}^{\mathrm{n}} \rightarrow \mathrm{R}$ and Borel set $\mathrm{B}$ on $\mathrm{R}$, one has Eq. 2:

$$
P\left[f\left(X_{1}, \ldots, X_{n}\right) \in B\right]=\int_{\Omega} P^{\omega}\left[f\left(\xi_{1}, \ldots, \xi_{n}\right) \in B\right] d P
$$

In what follows we shall use the following notations:

$$
\begin{aligned}
& \mathrm{T}_{\mathrm{n}}^{\omega}=\xi_{1}^{\omega}+\ldots+\xi_{\mathrm{n}}^{\omega} ; \\
& \mathrm{T}_{1, \mathrm{n}}^{\omega}=\xi_{1}^{\omega} 1\left(\left|\xi_{1}^{\omega}\right| \geq \mathrm{n}^{1 / \mathrm{r}}\right)+\ldots+\xi_{\mathrm{n}}^{\omega} 1\left(\left|\xi_{\mathrm{n}}^{\omega}\right| \geq \mathrm{n}^{1 / \mathrm{r}}\right) ; \\
& \xi_{2, \mathrm{n}}^{\omega}=\xi_{1}^{\omega} 1\left(\left|\xi_{1}^{\omega}\right|<\mathrm{n}^{1 / \mathrm{r}}\right)+\ldots+\xi_{\mathrm{n}}^{\omega} 1\left(\left|\xi_{\mathrm{n}}^{\omega}\right|<\mathrm{n}^{1 / \mathrm{r}}\right), \text { for } \mathrm{n} \geq 1
\end{aligned}
$$

According to (2) and the bounded convergence theorem, we have Eq. 3 :

$$
\sum_{n=1}^{\infty} n^{p / r-2} P\left[\left|S_{n}\right| \geq n^{1 / r}\right]=\int_{\Omega} \sum_{n=1}^{\infty} n^{p / r-2} P^{\omega}\left[\left|T_{n}^{\omega}\right| \geq n_{1 / r}\right] d P
$$

On one hand, using that $\sum_{\mathrm{n}=1}^{\infty} \mathrm{n}^{\mathrm{p} / \mathrm{r}-1} \leq \mathrm{Ck}^{\mathrm{p} / \mathrm{r}}$, we obtain:

$$
\begin{aligned}
& \sum_{\mathrm{n}=1}^{\infty} \mathrm{n}^{\mathrm{p} / \mathrm{r}-1} \mathrm{P}^{\omega}\left[\left|\xi_{1}^{\omega}\right| \geq \mathrm{n}^{1 / \mathrm{r}}\right] \leq \sum_{\mathrm{k}=1}^{\infty} \mathrm{P}^{\omega}\left[\mathrm{k}<\left|\xi_{1}^{\omega}\right|^{\mathrm{r}} \leq \mathrm{k}+1\right] \sum_{\mathrm{n}=1}^{\mathrm{k}} \mathrm{n}^{\mathrm{p} / \mathrm{r}-1} \\
& \leq \mathrm{C} \sum_{\mathrm{k}=1}^{\infty} \mathrm{k}^{\mathrm{p} / \mathrm{r}} \mathrm{P}^{\omega}\left[\mathrm{k}<\left|\xi_{1}^{\omega}\right|^{\mathrm{r}} \leq \mathrm{k}+1\right] \leq \mathrm{CE}^{\omega}\left(\left|\xi_{1}^{\omega}\right|^{\mathrm{p}}\right) \text { a.s }
\end{aligned}
$$

where, $\mathrm{E}^{\omega}$ denotes expectation under $\mathrm{P}^{\omega}$. Therefore:

$$
\begin{aligned}
& \int_{\Omega} \sum_{\mathrm{n}=1}^{\infty} \mathrm{n}^{\mathrm{p} / \mathrm{r}-2} \mathrm{P}^{\omega}\left[\left|\mathrm{T}_{1, \mathrm{n}}^{\omega}\right| \geq \mathrm{n}^{1 / \mathrm{r}}\right] \mathrm{dP} \leq \\
& \int_{\Omega} \sum_{\mathrm{n}=1}^{\infty} \mathrm{n}^{\mathrm{p} / \mathrm{r}-1} \mathrm{P}^{\omega}\left[\left|\xi_{1}^{\omega}\right| \geq \mathrm{n}^{1 / \mathrm{r}}\right] \mathrm{dP} \leq \mathrm{CE}\left|\mathrm{X}_{1}\right| \mathrm{p}<\infty
\end{aligned}
$$

On the other hand, by Lemma 2 we obtain:

$$
\begin{aligned}
& \sum_{n=1}^{\infty} n^{p / r-2} P^{\omega}\left[\left|T_{2, n}^{\omega}\right| \geq n^{1 / r}\right] \\
& \leq C \sum_{n=1}^{\infty} n^{-2} \sum_{k=1}^{n} E^{\omega}\left[\left(\left|\xi_{k}^{\omega}\right| \leq n^{1 / r}\right)\right]+C \sum_{n=1}^{\infty} n^{p / r-2} \\
& \exp \left\{-C \frac{n^{2 / r}}{n E^{\omega}\left[\left|\xi_{1}^{\omega}\right|^{p} 1\left(\left|\xi_{1}^{\omega}\right| \leq n^{1 / r}\right)\right]}\right\} \\
& \leq C \sum_{n=1}^{\infty} E^{\omega}\left[\left|\xi_{1}^{\omega}\right|^{p} 1\left(n-1<\left|\xi_{1}^{\omega}\right|^{r} \leq n\right)\right] \sum_{j=n}^{h} j^{-2} \\
& +C \sum_{n=1}^{\infty} n^{p / r-2} \exp \left\{-C \frac{n^{2 / r-1}}{E^{\omega}\left|\xi_{1}^{\omega}\right|^{2}}\right\} \\
& \leq C E^{\omega}\left(\left|\xi_{1}^{\omega}\right|^{p}\right)+C \sum_{n=1}^{\infty} n^{p / r-2} \exp \left\{-C n^{2 / r-1}\right\} a . s
\end{aligned}
$$

The latter series is convergent as $\mathrm{r}<2$. Therefore:

$$
\int_{\Omega} \sum_{\mathrm{n}=1}^{\infty} \mathrm{n}^{\mathrm{p} / \mathrm{r}-2} \mathrm{P}^{\omega}\left[\left|\mathrm{T}_{2, \mathrm{n}}^{\omega}\right| \geq \mathrm{n}^{1 / \mathrm{r}}\right] \mathrm{dP} \leq \mathrm{C}\left(\mathrm{E}\left|\mathrm{X}_{1}\right|^{\mathrm{p}}+1\right)<\infty
$$

Conclusion (1) now follows from (4) and (5) via (3).

\section{CONCLUSION}

It is very well known that we cannot allow $\mathrm{p}<1$ in the Baum-Katz estimate (1) for i.i.d. sequences; the following example shows that the same is true for exchangeable sequences. Consider $X_{n}=Y . \varepsilon_{n}$, where $\left\{\varepsilon_{n}\right\}_{n \geq 1}$ are i.i.d. and independent of a Cauchy random variable $\mathrm{Y}$, with $\mathrm{P}\left(\varepsilon_{1}=1\right)=\mathrm{P}\left(\varepsilon_{1}=-1\right)=1 / 2$. We have $\mathrm{E}\left|\mathrm{X}_{1}\right|^{\mathrm{p}}<\infty$ for all $0<\mathrm{p}<1$, but $\mathrm{E}\left|\mathrm{X}_{1}\right|=\infty$. As $\mathrm{X}_{1} \sim-\mathrm{X}_{1}$, we have:

$$
\frac{\mathrm{S}_{\mathrm{n}}}{\mathrm{n}}=\mathrm{Y} \cdot \frac{1}{\mathrm{n}} \sum_{\mathrm{i}=1}^{\mathrm{n}} \varepsilon_{\mathrm{i}} \rightarrow 0 \text { a.s }
$$

i.e., the strong law of large numbers holds for the exchangeable sequence $\left\{X_{n}\right\}_{n \geq 1}$. On the other hand:

$$
\begin{aligned}
& \sum_{\mathrm{n}=1}^{\infty} \mathrm{n}^{\mathrm{p} / \mathrm{r}-2} \mathrm{P}\left[\left|\mathrm{S}_{\mathrm{n}}\right| \geq \mathrm{n}^{1 / \mathrm{r}}\right]=\sum_{\mathrm{n}=1}^{\infty} \mathrm{n}^{\mathrm{p} / \mathrm{r}-2} \\
& \mathrm{P}\left(\left|\varepsilon_{1}+\ldots+\varepsilon_{\mathrm{n}}\right| \geq \mathrm{n}\right) \cdot \mathrm{P}\left(|\mathrm{Y}| \geq \mathrm{n}^{1 / \mathrm{r}-1}\right) \\
& \leq \mathrm{C} \sum_{\mathrm{n}=1}^{\infty} \mathrm{n}^{\mathrm{p} / \mathrm{r}-2} \int_{|\mathrm{x}| \geq \mathrm{n}^{1 / r-1}} \frac{1}{1+\mathrm{x}^{2}} \mathrm{dx}
\end{aligned}
$$

which diverges for all $\mathrm{p} \geq \mathrm{r}$ and $0<\mathrm{r}<2$.

\section{REFERENCES}

Chow, Y.S. and H. Teicher, 2003. Probability Theory: Independence, Interchangeability, Martingales. 3rd Edn., Springer, New York, ISBN-10: 0387406077, pp: 488. 
Etemadi, N. and M. Kaminski, 1996. Strong law of large numbers for 2-exchangeable random variables. Stat. Probab. Lett., 28: 245-250. DOI: 10.1016/0167-7152(95)00131-X

Etemadi, N., 2006. Convergence of weighted averages of random variables revisited. Proc. Am. Math. Soc., 134: 2739-2744. DOI: 10.1090/S0002-993906-08296-7

Petrov, V.V., 1995. Limit Theorems of Probability Theory: Sequences of Independent Random Variables. 1st Edn., Oxford University Press, New York, ISBN-10: 019853499X, pp: 292.
Rosalsky, A. and G. Stoica, 2010. On the strong law of large numbers for identically distributed random variables irrespective of their joint distributions. Stat. Probab. Lett., 80: 1265-1270. DOI: 10.1016/J.SPL.2010.04.005

Taylor, R.L. and T.C. Hu, 1987. On laws of large numbers for exchangeable random variables. Stoch. Anal. Appl., 5: 323-334. DOI: 10.1080/07362998708809120

Zhao, Y., 2004. The complete convergence for partial sums of interchangeable random variables. Chinese J. Appl. Probab. http://en.cnki.com.cn/Article_en/CJFDTOTALYYGN200402007.htm 